\title{
Parâmetros biológicos e tabela de vida de Tetranychus urticae (Acari: Tetranychidae) em cultivares de mamão
}

\author{
Biological parameters and life table of Tetranychus urticae (Acari: Tetranychidae) at papaya cultivars
}

\author{
Larissa Bernardino Moro ${ }^{\text {I* }}$ Ricardo Antônio Polanczyk ${ }^{\mathrm{II}}$ José Romário de Carvalho $^{\mathrm{I}}$ \\ Dirceu Pratissoli ${ }^{I}$ Cláudio Roberto Franco ${ }^{\text {III }}$
}

RESUMO

Este trabalho foi realizado para avaliar o desenvolvimento e reprodução de Tetranychus urticae em cultivares comerciais de mamão Carica papaya. Foram utilizadas cultivares do grupo "Formosa" ("Tainung 01 " $e$ "Calimosa") e do grupo "Solo" ("Golden" e "Sunrise"). Para iniciar o bioensaio, foi transferida uma fêmea fertilizada por disco de folha ( $n=50$ repetições) e retirado após um periodo de $12 \mathrm{~h}$, sendo avaliada a cada $12 \mathrm{~h}$, registrando-se o periodo de incubação, duração do estágio de imaturo, longevidade e fecundidade dos adultos e viabilidade desses estágios. Os resultados indicaram que as cultivares de mamão "Tainung 01", "Calimosa", "Sunrise" e "Golden" são bons hospedeiros para T. urticae. O parâmetro viabilidade não sofreu influência das cultivares em todas as fases de desenvolvimento avaliadas, apresentando valores superiores a 90\%. Não houve diferença estatística entre as cultivares nos parâmetros: período de preoviposição e viabilidade dos ovos. A cultivar "Tainung 01 " apresentou menor potencial hospedeiro, embora houvesse menor duração nos estágios de ovo, larva, protoninfa e ovoadulto. Nos parâmetros de tabela de vida e fertilidade, apresentou menores valores de $R, r_{m}$ e $\lambda$ e maior valor de Td. Entre as cultivares, o "Sunrise" apresentou um elevado potencial hospedeiro para T. urticae, pois essa cultivar proporcionou a maior produção de ovos por fêmea, maior longevidade das fêmeas, bem como a maior taxa de reprodução (maior $R_{o}, r_{m}$ e e menor valor de Td).

Palavras-chave: fitossanidade, ácaro-rajado, Carica papaya.

\begin{abstract}
The study was conducted to evaluate the Tetranichus urticae development and reproduction in commercial Carica papaya cultivars. The assayed cultivars belongs to the "Formosa" ("Tainung 01" and "Calimosa") and from the "Solo" ("Golden" and "Sunrise") groups. A fertilized female was transferred to a leaf dish ( $n=50$ repetitions) and was removed after a period of 12 hours and evaluations were done every 12 hours recording the incubation period, length of immature stage, longevity and fecundity of adults and viability of these stages. The cultivars of papaya, "Tainung 01", "Calimosa", "Sunrise" and "Golden" are adequate hosts for T. urticae. The recorded viability above 90\% was not influenced by the different cultivars during all the development phases. There was not statistic difference among the cultivars in the parameters: pre-ovopositional period, and egg viability. Mites assayed in "Tainung 01" cultivar showed a shorter duration of the egg, larva, protonymph and egg-adult. At life table parameters and fertility, presented smaller $R_{o}, r_{m}$ and and larger Td. "Sunrise" is the cultivar most adequate for $\mathbf{T}$. urticae development, considering that mites assayed in this cultivar showed a larger production of eggs per female, longer female longevity, and higher reproduction rate (larger $R_{o}, r_{m}$ and and smaller Td).
\end{abstract}

Key words: phytosanity, two-spotted spider mite, Carica papaya.

\section{INTRODUÇÃO}

O Brasil tem se destacado como segundo maior produtor e exportador de mamão Carica papaya

\footnotetext{
IUniversidade Federal do Espírito Santo (UFES), Centro de Ciências Agrárias (CCA), Departamento de Fitotecnia, Alto Universitário, s/nº, CP 16, Guararema, 29500-000, Alegre, ES, Brasil. E-mail: larissamoro@hotmail.com. *Autor para correspondência.

"Universidade Estadual Paulista "Júlio de Mesquita Filho" (UNESP), Faculdade de Ciências Agrárias e Veterinárias (FCAV), Departamento de Fitossanidade, Jaboticabal, SP, Brasil.

IIIUniversidade do Estado de Santa Catarina (UDESC), Centro de Ciências Agroveterinárias (CAV), Departamento de Agronomia, Lages, SC, Brasil.
} 
(Linneu, 1753), com oferta, entre os anos de 2006-2008, em torno de 20 e $14 \%$ da produção mundial, respectivamente (AGRAFNP, 2011). No mercado brasileiro, as variedades mais cultivadas pertencem ao grupo Solo, também conhecidos como "mamão Havaí" ou "mamão Papaya". Desse grupo, destacam-se as cultivares "Sunrise" Solo e "Golden", e os híbridos do grupo Formosa, cultivar "Tainung 01". Essas cultivares foram provenientes do Havaí (EUA) e China introduzidos no Brasil no início da década de 1970. Em 2003, foi lançado o primeiro híbrido brasileiro do grupo Formosa, chamado de UENF-Caliman 01 ("Calimosa") (MARTINS \& COSTA, 2003; RUGGIERO et al., 2003; FERREGUETTI, 2003). No Brasil, a zona de cultivo mais relevante para o mamoeiro localiza-se no sul da Bahia e norte do Espírito Santo, regiões que respondem por cerca de $85 \%$ da produção nacional, onde prevalecem condições de grande insolação e temperaturas entre 22 e $28^{\circ} \mathrm{C}$ (SOUZA et al., 2000;AGRAFNP, 2011), embora seja num clima favorável para o desenvolvimento de pragas, podendo-se destacar os ácaros tetraniquídeos (MARTINS \& MARIN, 1998; CULIK, 2003).

O tetraniquídeo Tetranychus urticae (KOCK, 1836), comumente conhecido como ácarorajado, é uma das pragas-chave do mamoeiro $\boldsymbol{C}$. papaya, infestando a cultura durante todo o ano, com maior severidade nos períodos de baixa precipitação pluviométrica e temperaturas elevadas. Quando ocorre alta infestação, há redução da área fotossintética por causa da necrose de tecidos foliares e queda prematura de folhas, principalmente as folhas maduras, consequentemente, compromete a produtividade da planta, além de prejudicar a qualidade dos frutos por causa da maior exposição aos raios solares (MARIN et al., 1995; MARTINS \& COSTA, 2003; MORAES \& FLECHTMANN, 2008).

O desenvolvimento de T. urticae pode ser influenciado pela espécie de planta utilizada como hospedeira, assim como pode ocorrer variação intraespecífica, em função dos diferentes genótipos utilizados nos cultivos comerciais (KRIPS et al., 1998; GRECO et al., 2006; VAN DEN BOOM et al., 2003). No Brasil, há estudos que comparam o desenvolvimento de T. urticae em diferentes espécies e cultivares de plantas de importância econômica, como em algodoeiro Gossypium hirsutum (SILVA et al., 1985a; ESTEVES FILHO et al., 2010), morangueiro Fragaria x ananassa (LOURENÇÃ̃ et al., 2000), tomateiro Lycopersicon spp. (MARUYAMA et al., 2002) e gérbera Gerbera jamesonii (SILVA et al., 2009). Esses estudos são importantes para a confecção de tabelas de vida e fertilidade que agregam informações sobre parâmetros reprodutivos e de mortalidade, permitindo a compreensão da dinâmica populacional de determinadas espécies de insetos ou ácaros (SILVEIRA NETO et al., 1976) e contribuindo para subsidiar um manejo fitossanitário mais eficiente de pragas.
Frente a isso, o presente trabalho teve como objetivo avaliar a biologia e reprodução de T. urticae em cultivares comerciais de $\boldsymbol{C}$. papaya para a confecção da tabela de vida.

\section{MATERIAIS E MÉTODOS}

A pesquisa foi conduzida no Núcleo de Desenvolvimento Científico e Tecnológico em Manejo Fitossanitário (NUDEMAFI) do Centro de Ciências Agrárias da Universidade Federal do Espírito Santo (CCA/UFES), Alegre-ES.

\section{Coleta e criação de T. urticae em laboratório}

A população de $\boldsymbol{T}$. urticae, utilizada nos experimentos, foi coletada em mamoeiros em LinharesES em 2006 e mantida em condições de laboratório sobre plantas de feijão-de-porco Canavalia ensiformis (L.) D.C. sob condições controladas $\left(25 \pm 2^{\circ} \mathrm{C}, 70 \pm 20 \%\right.$ de UR e fotofase de 14 horas). Essa criação foi denominada criação estoque.

\section{Cultivares de C. papaya}

Foram utilizadas quatro cultivares de mamão, sendo dois do grupo "Formosa" ("Tainung 01 " e "Calimosa") e dois do grupo "Solo" ("Golden" e "Sunrise"), representando as cultivares mais utilizadas em lavouras comerciais. As mudas foram plantadas em

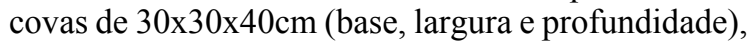
adubadas com dois litros de esterco bovino e $150 \mathrm{~g}$ de calcário em cada cova, seguindo as recomendações de PREZOTTI et al. (2007).

Biologia e tabela de vida e fertilidade de T. urticae em C. papaya

Foram seccionados discos de dois centímetros de diâmetro de folhas de cada cultivar em estágio adulto, sendo acondicionados, com a face abaxial (inferior) voltada para cima, em placas de Petri de nove centímetros de diâmetro, sobre uma camada de algodão umedecida diariamente com água destilada para manter a turgidez dos discos de folha e impedir a fuga dos ácaros. Uma fêmea de T. urticae, proveniente da criação estoque, fecundada, foi transferida com o auxílio de um pincel fino para cada disco de folha de modo que ocorresse oviposição. As fêmeas foram retiradas dos discos de folha após um período de 12 horas, sendo deixados dois ovos/disco de folha e, após a eclosão da larva, foi acompanhado apenas um indivíduo/disco de folha, totalizando 50 discos $(n=50$ repetições) para cada cultivar. Os discos de folha foram trocados a cada três dias. $\mathrm{O}$ experimento permaneceu em câmara climatizada à temperatura de $26 \pm 1^{\circ} \mathrm{C}, 70 \pm 10 \%$ de UR e fotofase de 12 horas.

As avaliações foram feitas a cada 12 horas sendo os seguintes parâmetros observados: duração e viabilidade dos estágios de imaturo (larva, protoninfa e deutoninfa); longevidade e fecundidade dos adultos. 
O período de incubação e viabilidade dos ovos foi avaliado a partir dos ovos ovipositados durante as três avaliações iniciais do período de oviposição pelas fêmeas em cada cultivar. A partir desses dados, foram confeccionadas as tabelas de vida e fertilidade, calculando os valores de $R_{o}$ (taxa líquida de reprodução), $r_{m}$ (taxa intrínseca de crescimento populacional), (razão finita de aumento) e $T$ (duração média de uma geração).

Análise estatística

Os dados foram submetidos à análise de variância e as médias comparadas pelo teste de Tukey a 5\% de probabilidade. Os parâmetros da tabela de vida e fertilidade foram estimados pelo método Jackknife e comparados pelo teste $t$ de student bilateral a $5 \%$ de probabilidade, utilizando o pacote estatístico SAS System for Windows versão 8.0 (MAIA et al., 2000).

\section{RESULTADOS E DISCUSSÃO}

\section{Biologia de T. urticae em C. papaya}

A duração da fase de ovo apresentou diferença significativa $\left(\mathrm{F}_{3,200}=9,13 ; \mathrm{P}<0,0001\right)$ entre a cultivar "Tainung 01 " (4,0 dias) e as cultivares "Calimosa", "Sunrise" e "Golden", variando entre 4,2 e 4,3 dias (Figura 1A). Na duração das fases de larva e

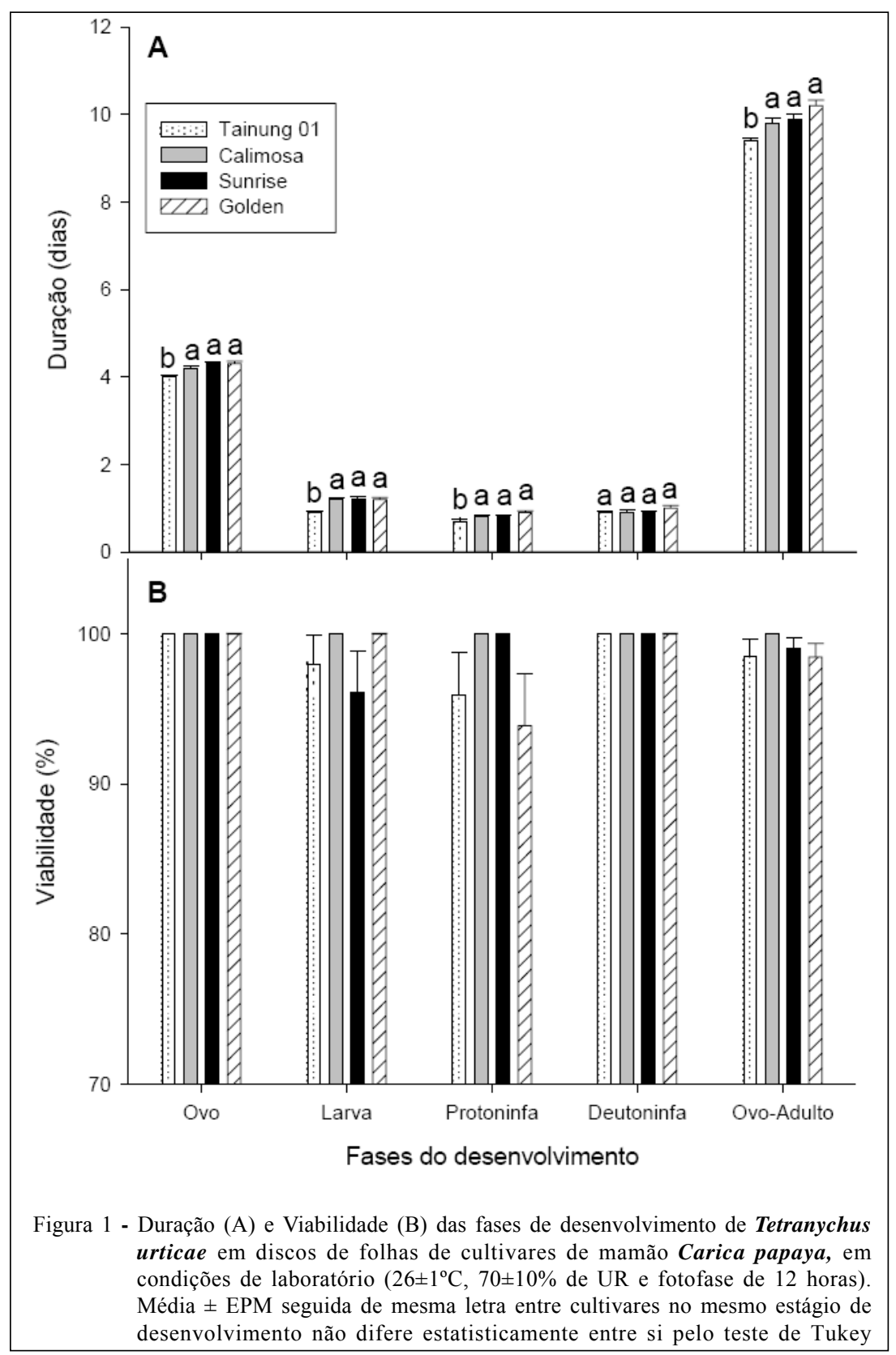

Ciência Rural, v.42, n.3, mar, 2012. 
protoninfa, também houve diferenças significativas $\left(\mathrm{F}_{3,200}=4,20 ; \mathrm{P}=0,0066\right.$ para larva e $\mathrm{F}_{3,190}=4,81 ; \mathrm{P}=0,003$ para protoninfa), sendo os menores períodos desses estágios de desenvolvimento observados na cultivar "Tainung 01" (0,9 e 0,7 dias, respectivamente). Os valores de duração das fases de larva nas demais cultivares foram de 1,2 dias, enquanto a fase de protoninfa oscilou entre 0,8 e 0,9 dias (Figura 1A). O período correspondente à fase de deutoninfa não apresentou variação significativa entre as cultivares. Por sua vez, a duração do período ovo-adulto diferiu significativamente entre a cultivar "Tainung 01 " $(9,4$ dias) e demais cultivares ( 9,8 e 10,2 dias) $\left(\mathrm{F}_{3,164}=9,44\right.$; $\mathrm{P}<0,0001$ ) (Figura 1A). A espécie da planta hospedeira é um fator biótico importante na capacidade de crescimento populacional das espécies de ácaros. Segundo SILVA et al. (1985a) o período ovo-adulto de T. urticae entre cultivares de algodão (IAC-17, IAC-18 e IAC-19) variaram entre 10,6 e 10,7 dias. No trabalho de ESTEVES FILHO et al. (2010) em cultivares de algodão BT (Acala DP 90B), lançado recentemente no mercado, e não-BT (Acala DP 90), o período ovo-adulto foi entre 8,9 e 9,3 dias. Embora esses autores não tenham observado diferença nesse parâmetro biológico entre as cultivares de algodão, observa-se que foram diferentes dos resultados observados no presente trabalho. Embora seja em outra cultivar de algodão (CNPA ITA 90), SILVA (2002) observou, para o ácarovermelho Tetranychus ludeni, período ovo-adulto entre 13,3 e 12,6 dias para fêmeas e macho, respectivamente, e que pode variar entre 20,8 e 8,5 dias em função da temperatura que, no estudo, variou entre 20 e $30^{\circ} \mathrm{C}$. Dessa forma, ressalta-se a importância da relação ácaro, planta e ambiente nos estudos de bioecologia para compreender a dinâmica populacional de uma espécie de ácaro no ecossistema agrícola.

No parâmetro viabilidade dos estágios imaturos (larva, protoninfa, deutoninfa e período ovoadulto), não houve influência das cultivares no desenvolvimento de T. urticae. A viabilidade do período ovo-adulto foi entre 94,1 e 100,0\% (Figura 1-B). Assim como a viabilidade dos ovos ovipositados pelas fêmeas alimentadas com as cultivares de mamão que variou entre 68,7 e 77,4\% (Tabela 1). A viabilidade de ovos de T. urticae observada nesse trabalho foi inferior à viabilidade em outros hospedeiros. Por exemplo, SILVA et al. (2009) relataram, em folhas de gérbera, a viabilidade de ovos de 97\%, em cultivares de algodoeiro, a viabilidade foi entre 84 e 87\% (SILVA et al., 1985a) e, em cultivares de algodão BT e não-BT, a viabilidade foi entre 94 e 95\% (ESTEVES FILHO et al., 2010).

Não houve diferença estatística entre as cultivares no período de pre-oviposição de fêmeas de T. urticae entre as cultivares de mamoeiro, de 1,4 a 1,6 dias. No parâmetro longevidade de fêmeas e machos, houve diferença significativa $\left(\mathrm{F}_{3,152}=4,48 ; \mathrm{P}=0,005\right.$ para fêmeas e $\mathrm{F}_{3,40}=5,17 ; \mathrm{P}=0,0041$ para machos). As cultivares "Sunrise" e "Calimosa" proporcionaram maior longevidade de fêmeas, 16,9 e 14,1 dias, respectivamente, não diferindo entre si, enquanto a cultivar "Tainung 01 " foi a dieta que proporcionou menor longevidade de fêmeas (11,9 dias), mas não diferindo das cultivares "Golden" e "Calimosa" (13,0 e 14,1 dias respectivamente). Embora a cultivar "Tainung 01 " tenha sido a mais favorável ao desenvolvimento de T. urticae durante os estágios de imaturos, esse resultado não se repetiu na longevidade de fêmeas. Enquanto no parâmetro número de ovos por fêmea foram observadas diferenças estatísticas $\left(\mathrm{F}_{3,128}=4,14\right.$; $\mathrm{P}=0,0077$ ), com destaque para a cultivar "Sunrise" com maior número de ovos (33,9 ovos por fêmea), não houve diferença das cultivares "Tainung 01" e "Calimosa", indicando que essas cultivares foram adequados ao desenvolvimento de T. urticae (Tabela 1).

A qualidade nutricional da planta hospedeira é fator determinante para o desempenho de um herbívoro, pois se sabe que alterações na fisiologia e bioquímica da planta afetam diretamente o

Tabela 1 - Parâmetros biológicos do ciclo de vida de Tetranychus urticae em discos de folha de cultivares de mamão Carica papaya, em condições de laboratório $\left(26 \pm 1^{\circ} \mathrm{C}, 70 \pm 10 \%\right.$ de UR e fotofase de 12 horas).

\begin{tabular}{|c|c|c|c|c|c|c|}
\hline Cultivares & Pré-oviposição & $\begin{array}{l}\text { Número de } \\
\text { ovos/fêmea }\end{array}$ & $\begin{array}{l}\text { Viabilidade dos } \\
\text { ovos }\end{array}$ & $\begin{array}{l}\text { Longevidade de } \\
\text { fêmea }\end{array}$ & $\begin{array}{l}\text { Longevidade de } \\
\text { macho }\end{array}$ & $\begin{array}{l}\text { Ciclo total de } \\
\text { fêmea }\end{array}$ \\
\hline “Tainung 01” & $1,6 \pm 0,12 \mathrm{a}$ & $30,5 \pm 2,27 \mathrm{ab}$ & $71,4 \pm 4,22 \mathrm{a}$ & $11,9 \pm 1,06 \mathrm{~b}$ & $4,7 \pm 0,76 \mathrm{c}$ & $23,9 \pm 1,05 a b$ \\
\hline “Calimosa” & $1,6 \pm 0,11 \mathrm{a}$ & $28,6 \pm 1,76 \mathrm{ab}$ & $68,7 \pm 4,67 \mathrm{a}$ & $14,1 \pm 0,91 \mathrm{ab}$ & $20,2 \pm 2,78 \mathrm{a}$ & $24,1 \pm 0,80 \mathrm{ab}$ \\
\hline "Sunrise" & $1,4 \pm 0,07 \mathrm{a}$ & $33,9 \pm 2,09 \mathrm{a}$ & $74,6 \pm 4,45 \mathrm{a}$ & $16,9 \pm 1,03 \mathrm{a}$ & $8,8 \pm 1,77$ bc & $27,0 \pm 0,97 \mathrm{a}$ \\
\hline “Golden” & $1,5 \pm 0,09 \mathrm{a}$ & $25,4 \pm 1,98 \mathrm{~b}$ & $77,4 \pm 4,34 \mathrm{a}$ & $13,0 \pm 1,11 b$ & $15,4 \pm 2,71 \mathrm{ab}$ & $23,6 \pm 0,95 b$ \\
\hline
\end{tabular}

Média \pm EPM seguida de mesma letra na linha não difere estatisticamente entre si pelo teste de Tukey $(\mathrm{P}>0,05)$.

Ciência Rural, v.42, n.3, mar, 2012. 
desenvolvimento de um herbívoro (AWMACK \& LEATHER, 2002). Resultados semelhantes ao presente estudo foram observados por MARUYAMA et al. (2002) entre cultivares de tomateiro. Eles foram classificados segundo o índice de preferência para oviposição como deterrentes, porque houve 0 a 3 ovos/ folíolo, enquanto em plantas consideradas estimulantes houve entre 36 e 50 ovos/folíolo. Diferenças entre espécies de plantas também constituem fator importante para sobrevivência de uma espécie de ácaros no mesmo ecossistema, principalmente para espécies polífagas como o T. urticae, proporcionando muitas vezes a sua permanência durante entre safras. FADINI et al. (2004) observaram, em folhas de morangueiro, 58 ovos/fêmea, SILVA et al. (2009), em gérbera, encontraram 41 ovos/fêmea (fêmeas nãoacasaladas) e ESTEVES FILHO et al. (2010), em algodoeiro, relataram 180 ovos/fêmea, valores superiores ao número de ovos obtidos no presente estudo. GRECO et al. (2006) também relataram diferenças entre espécies de plantas hospedeiras na aceitação de T. urticae. Esses autores observaram que a fecundidade desse ácaro em morango foi 5 a 7 vezes maior do que em salsinha Petroselinum sativum, cebola Allium cepa e alho-porró Allium porrum. Outras espécies de tetraniquídeos também apresentam grande plasticidade genética, como observado por SOUZA et al. (2010). Esses autores relataram uma diferença na produção de ovos de Tetranychus mexicanus quando criado em diferentes anonáceas, sendo observados 99 ovos/fêmea em graviola Annona muricata, 65 ovos/ fêmeas em fruta-do-conde Annona squamosa e 39 ovos/fêmea em araticum Annona coriaceae.

$\mathrm{Na}$ longevidade de machos, as cultivares também proporcionaram diferenças estatísticas entre si, com longevidade na cultivar "Tainung 01 " (4,7 dias) menor em relação as demais, sendo semelhante estatisticamente apenas a cultivar "Sunrise" ( 8,8 dias) (Tabela 1). Valores baixos de longevidade de macho, quando comparado à longevidade de fêmeas, também foram observados em outros trabalhos, como entre cultivares de algodão (SILVA et al., 1985a) e inclusive com outras espécies de tetraniquídeos, como T. Iudeni em algodão (SILVA, 2002).

Tabela de vida e fertilidade de T. urticae em C. papaya A taxa líquida de reprodução $\left(\mathrm{R}_{\mathrm{o}}\right)$ que corresponde à contribuição média de cada fêmea para a geração seguinte, expresso em número de descendentes fêmeas por fêmea, foi influenciada significativamente pelas cultivares de mamão $\left(\mathrm{F}_{3,153}=3,23 ; \mathrm{P}=0,0243\right)$. $\mathrm{O}$ maior $\mathrm{R}_{\mathrm{o}}$ foi observado na cultivar "Sunrise" (106,7 fêmeas/fêmea), enquanto entre as cultivares "Tainung 01 ", "Calimosa" e "Golden" não houve diferenças estatísticas, variando entre 76,5 e 81,5 fêmeas/fêmea (Tabela 2). Esse resultado corrobora os dados observados nos parâmetros número de ovos/fêmea e longevidade de fêmeas que foram maiores na cultivar "Sunrise" do que nas demais cultivares, principalmente quando comparado com "Tainung 01" no parâmetro longevidade de fêmeas, que contribui no fator sobrevivência utilizado no cálculo de $\mathrm{R}_{\mathrm{o}}$. Os valores de $\mathrm{R}_{\mathrm{o}}$, como são estimados a partir de valores de reprodução e mortalidade a planta hospedeira, terão importante influência no crescimento populacional do ácaro. Segundo KASAP (2004), houve diferença no $\mathrm{R}_{0}$ de $T$. urticae entre cultivares de macieira Malus domestica, observando oscilação entre 64 e 92 fêmeas/fêmea. SILVA et al. (1985b) também observaram valores de $\mathrm{R}_{\mathrm{o}}$ diferentes entre cultivares de algodão a $26^{\circ} \mathrm{C}$ (40 a 48 fêmeas/fêmea). Esse parâmetro tem influência direta na taxa de intrínseca de aumento $\left(\mathrm{r}_{\mathrm{m}}\right)$ que não foi diferente entre as cultivares "Sunrise", "Golden" e "Calimosa", mas diferiram do "Tainung 01" (Tabela 2). HELLE \& SABELIS (1985) agruparam valores de $r_{m}$ citados na literatura e verificaram uma amplitude entre 0,22 e 0,28 fêmeas fêmea ${ }^{-1} \mathrm{dia}^{-1}$, sendo essas extremidades influenciadas pela resistência da planta à

Tabela 2 - Tabela de vida e fertilidade de Tetranychus urticae em discos de folha de cultivares de mamão Carica papaya, em condições de laboratório $\left(26 \pm 1^{\circ} \mathrm{C}, 70 \pm 10 \%\right.$ de UR e fotofase de 12 horas $)$.

\begin{tabular}{|c|c|c|c|c|c|}
\hline \multirow{2}{*}{ Cultivares } & \multirow[b]{2}{*}{$\mathrm{R}_{\mathrm{o}}$} & \multirow[b]{2}{*}{$\mathrm{T}$} & \multirow[b]{2}{*}{$\mathrm{Td}$} & \multirow[b]{2}{*}{$\mathrm{r}_{\mathrm{m}}$} & \multirow[b]{2}{*}{$\Lambda$} \\
\hline & & & & & \\
\hline "Tainung 01" & $81,5 \pm 8,64 b$ & $15,9 \pm 0,29 \mathrm{a}$ & $2,49 \pm 0,046 \mathrm{a}$ & $0,28 \pm 0,005 \mathrm{~b}$ & $1,32 \pm 0,006 \mathrm{~b}$ \\
\hline "Calimosa" & $76,5 \pm 6,61 b$ & $15,2 \pm 0,24 \mathrm{~b}$ & $2,43 \pm 0,051 \mathrm{ab}$ & $0,28 \pm 0,005 \mathrm{a}$ & $1,33 \pm 0,007 \mathrm{a}$ \\
\hline "Sunrise" & $106,7 \pm 8,53 \mathrm{a}$ & $16,0 \pm 0,16 \mathrm{a}$ & $2,37 \pm 0,041 \mathrm{~b}$ & $0,29 \pm 0,005 \mathrm{a}$ & $1,34 \pm 0,006 \mathrm{a}$ \\
\hline "Golden" & $76,7 \pm 8,20 b$ & $15,5 \pm 0,29 \mathrm{ab}$ & $2,47 \pm 0,041 \mathrm{a}$ & $0,28 \pm 0,004 \mathrm{ab}$ & $1,32 \pm 0,006 \mathrm{a}$ \\
\hline
\end{tabular}

Média \pm EPM seguida pela mesma letra na linha não difere estatisticamente entre si pelo método estimado de Jackknife.

Parâmetros de tabela de vida e fertilidade: $\mathrm{R}_{\mathrm{o}}=$ Taxa líquida de reprodução; $\mathrm{T}=$ Duração média de uma geração em dias; $\mathrm{Td}=\mathrm{Tempo}$ de duplicação em dias; $r_{m}=$ Taxa intrínseca de aumento; e $\lambda=$ Razão finita de aumento. 
T. urticae. Segundo KRIPS et al. (1998), os valores de $\mathrm{r}_{\mathrm{m}}$ de $\boldsymbol{T}$. urticae em cultivares de gérbera resistente e suscetível foram de 0,21 e 0,26 , respectivamente. Ambos os parâmetros $\left(\mathrm{R}_{\mathrm{o}} \mathrm{e} \mathrm{r}_{\mathrm{m}}\right)$ influenciam no tempo médio em dias entre gerações $(\mathrm{T})$ e no tempo em dias necessário para duplicação do tamanho inicial de uma população (Td). Os valores de T de T. urticae nas cultivares "Tainung 01", "Sunrise" e "Golden" não diferiram estatisticamente, corroborando o parâmetro duração do ciclo de fêmea (Tabela 1), já que não houve diferenças entre essas cultivares, exceto entre "Sunrise" e "Golden". Enquanto o Td na cultivar "Sunrise" foi menor, a população duplicou de tamanho a cada 2,37 dias e, na "Tainung 01", a cada 2,49 dias. Enquanto a razão finita de aumento $(\lambda)$ representa um fator de multiplicação da população dentro de um intervalo de tempo, podendo ser expressa como taxa de crescimento diário em porcentagem. A maior taxa de crescimento diário foi observada na cultivar "Sunrise" com 34\%, não diferindo das cultivares "Calimosa" e "Golden", mas houve diferença com a "Tainung 01" (menor crescimento com $32 \%$ ).

Embora as cultivares de mamão fossem adequadas ao desenvolvimento de T. urticae, baseado nos parâmetros biológicos de duração dos estágios de desenvolvimento e da tabela de vida e fertilidade, observa-se que a cultivar "Tainung 01 " proporcionou menor duração do ciclo ovo-adulto. No entanto, nos parâmetros de $\mathrm{R}_{\mathrm{o}}, \mathrm{Td}, \mathrm{r}_{\mathrm{m}} \mathrm{e}$, nessa cultivar, foi observado menor desempenho de T. urticae comparado às demais cultivares, principalmente quando comparado à cultivar "Sunrise", que possibilitou maior potencial de crescimento populacional desse ácaro. Essa condição pode ser consequência de características intrínsecas das cultivares, como composição nutricional do hospedeiro ou ainda relacionada a fatores de defesa física, morfológica ou química da planta contra herbivoria.

Segundo MARUYAMA et al. (2002), a ausência ou menor quantidade de tricomas glandulares (fator de resistência morfológica) está relacionada à menor oviposição de $\boldsymbol{T}$. urticae em diferentes genótipos de tomateiro. Segundo KONNO et al. (2004), folhas de C. papaya apresentam no látex produzido pelas folhas uma protease, a papaína, que confere defesa contra o ataque de herbívoros, inclusive de insetos polífagos, reduzindo sua taxa de crescimento nesse hospedeiro. Dessa forma, estudos futuros poderiam explorar uma possível diferença no teor dessa enzima em cultivares de mamão que possa explicar diferenças da herbivoria causada pelo T. urticae, assim como de outros organismos fitófagos.

No entanto, sabe-se que, no processo de seleção da planta hospedeira pelos ácaros, embora limitada pela sua capacidade de dispersão, ocorre preferência por plantas adequadas ao seu desenvolvimento, mas que, após algumas gerações sobre um novo hospedeiro, pode ocorrer a sua adaptação. A redução nos valores de $\mathrm{r}_{\mathrm{m}}$ de Tetranychus spp. sobre plantas menos favoráveis ao seu desenvolvimento pode ocorrer porque o ácaro necessita alocar energia, por exemplo, para destoxificação causada por compostos secundários produzidos pela planta hospedeira ou para evitar plantas com esses compostos secundários que são tóxicos (HELLE \& SABELIS, 1985; GRECO et al., 2006). No entanto, KRIPS et al. (1998) observaram que, em cultivares de gérbera resistentes ao T. urticae, mesmo após 10 gerações desse ácaro, o valor de $\mathrm{r}_{\mathrm{m}}$ se manteve baixo quando comparado à cultivar suscetível.

Essas diferenças na taxa de crescimento populacional terão reflexos nos danos causados à cultura e na tomada de decisão pelo controle. Segundo LOURENÇÃO et al. (2000), nas cultivares de morangueiro que apresentavam as menores notas de sintomas de ataque causado por T. urticae, dentro de uma escala de nota que variava entre plantas sem sintomas até plantas mortas, foram as cultivares que apresentaram as menores infestações. Portanto, em estudos da aceitação de T. urticae, a planta hospedeira (espécies e cultivares) é fundamental para o manejo integrado de pragas, seja para fornecer informações da bioecologia desse ácaro a fim de aprimorar estratégias de monitoramento. Isso visa a contribuir com os programas de melhoramento genético para obtenção de cultivares resistentes, possibilitando, por exemplo, a integração da resistência de plantas e outros métodos de controle. Um exemplo disso é o controle biológico com uso de ácaros predadores para contribuir na manutenção da densidade populacional desse ácaro abaixo do nível de dano econômico.

\section{CONCLUSÃO}

Baseado nos parâmetros de tabela de vida $\mathrm{e}$ fertilidade de T. urticae em C. papaya, a cultivar "Tainung 01 " foi menos favorável ao desenvolvimento de T. urticae, enquanto as cultivares "Sunrise", "Golden" e "Calimosa" foram as mais favoráveis ao desenvolvimento desse ácaro, quando comparados os parâmetros $R_{o}, r_{m}, T d e$. 


\section{REFERÊNCIAS}

AGRAFNP. AgrianUAL 2011: Anuário da Agricultura Brasileira. São Paulo: AgraFNP, 2011. 482p.

AWMACK, C.S.; LEATHER, S.R. Host plant quality and fecundity in herbivorous insects. Annual Review of Entomology, v.47, p.817-844, 2002.

CULIK, M.P. et al. Índice de artrópodes pragas do mamoeiro (Carica papaya L.). Vitória: INCAPER, 2003. 48p. (Documento, 121).

ESTEVES FILHO, A.B. et al. Biologia comparada comportamento de Tetranychus urticae Koch (Acari: Tetranychidae) e Phytoseiulus macropilis (Banks) (Acari: Phytoseiidae) em Algodoeiro Bollgard ${ }^{\mathrm{TM}}$ e Isolinha nãoTransgênica. Neotropical Entomology, v.39, n.3, p.338-344, 2010. Disponível em: <http://www.scielo.br/pdf/ne/v39n3/ v39n3a05.pdf>. Acesso em: 05 set. 2011.

FADINI, M.A.M. et al. Herbivoria de Tetranychus urticae Koch (Acari: Tetranychidae) induz defesa direta em morangueiro? Neotropical Entomology, v.33, n.3, p.293-297, 2004 Disponível em: <http://www.scielo.br/pdf/ne/v33n3/ v33n3a03.pdf>. Acesso em: 05 set. 2011.

FERREGUETTI, G.A. Caliman 01- o primeiro híbrido de mamão formosa brasileiro. In: MARTINS, D. dos S. (ed.). Papaya Brasil: qualidade do mamão para o mercado interno. Vitória, ES: INCAPER, 2003. p.211-218.

GRECO, N.M. et al. Host-plant acceptance and performance of Tetranychus urticae (Acari: Tetranychidae). Journal of Applied Entomology. v.130, n.1, p.32-36, 2006

HELlE, W.; SABELIS, M.W. Spider mites. Their biology, natural enemies and control. Amsterdam: Elsevier, 1985. 1Av.

KASAP, I. Effect of apple cultivar and of temperature on the biology and life table parameters of the twospotted spider mite Tetranychus urticae. Phytoparasitica, v.32, n.1, p.73-82, 2004 Disponível em: <http://www.springerlink.com/content/ g173p27430446081/fulltext.pdf>. Acesso em: 09 set. 2011.

KRIPS, O.E. et al. Intrinsic rate of population increase of the spider mite Tetranychus urticae on the ornamental crop gerbera: intraspecific variation in host plant and herbivore. Entomologia Experimentalis et Applicata, v.89, p.159-168, 1998.

KONNO et al. Papain protects papaya trees from herbivorous insects: role of cysteine proteases in latex. Plant Journal, v.37, p.370-378, 2004 .

LOURENÇÃO, A.L. et al. Resistência de morangueiros a Tetranychus urticae Koch (Acari: Tetranychidae). Anais da Sociedade Entomológica do Brasil, v.29, n.2, p.339-346, 2000. Disponível em: < http://www.scielo.br/pdf/aseb/v29n2/ v29n2a16.pdf>. Acesso em: 05 set. 2011.

MAIA, A. de H.N. et al. Statistical Inferences on associated lifetable parameters using jackknife technique: computational aspects. Journal of Economic Entomology, v.93, n.2, p.511518, 2000.

MARIN, S.L.D. et al. Recomendações para a cultura do mamoeiro dos grupos Solo e Formosa no Estado do Espírito Santo. 4.ed. rev. e ampl. Vitória, ES: EMCAPA, 1995. 57p. (EMCAPA. Circular Técnica, 3).
MARUYAMA, W.I. et al. Resistência de genótipos de tomateiro ao ácaro rajado. Horticultura Brasileira, v.20, n.3, p.480484, 2002. Disponível em: <http://www.scielo.br/pdf/hb/v20n3/ 14474.pdf $>$. Acesso em: 05 set. 2011.

MARTINS, D.S.; COSTA, A.F.S. A cultura do mamoeiro: tecnologias de produção. Vitória, ES: Incaper, 2003. 497p.

MARTINS, D. dos S.; MARIN, S.L.D. Pragas do mamoeiro. In: BRAGA SOBRINHO, L.B. et al. (Eds.). Pragas de fruteiras tropicais de importância agroindustrial. Brasília: EMBRAPA CNPAT, 1998. p.143-153.

MORAES, G.J.; FLECHTMANN, C.H.W. Manual de acarologia: acarologia básica e ácaros de plantas cultivadas no Brasil. Ribeirão Preto: Holos, 2008. 308p.

PREZOTTI, L.C. et al. Manual de recomendação de calagem e adubação para o estado do Espírito Santo - $5^{\text {a }}$ aproximação. Vitória: SEEA/INCAPER/CEDAGRO, 2007. 305p.

RUGGIERO, C. et al. Panorama da cultura do mamão no Brasil e no mundo: situação atual e tendências. In: MARTINS, D. dos S. (Ed.). Papaya Brasil: qualidade do mamão para o mercado interno. Vitória, ES: INCAPER, 2003. p.13-34.

SILVA, C.A.D. Biologia e exigências térmicas do ácaro-vermelho (Tetranychus ludeni Zacher) em folhas de algodoeiro. Pesquisa Agropecuária Brasileira, v.37, n.5, p.573-580, 2002. Disponível em: <http://www.scielo.br/pdf/pab/v37n5/9525.pdf>. Acesso em: 05 set. 2011

SILVA, E.A. et al. Tetranychus urticae (Acari: Tetranychidae) on Gerbera jamesonii Bolus and Hook (Asteraceae). Braziliam Journal of Biology, v.69, n.4, p.1121-1125, 2009. Disponível em: <http://www.scielo.br/pdf/bjb/v69n4/v69n4a16.pdf>. Acesso em: 05 set. 2011.

SILVA, M.A. et al. Biologia comparada de Tetranychus urticae em cultivares de algodoeiro: ciclo biológico. Pesquisa Agropecuária Brasileira, v.20, n.7, p.741-748, 1985a

SILVA, M.A. et al. Biologia comparada de Tetranychus urticae em cultivares de algodoeiro: tabela de vida de fertilidade. Pesquisa Agropecuária Brasileira, v.20, n.9, p.1015-1019, 1985b.

SILVEIRA NETO, S. et al. Manual de ecologia dos insetos. São Paulo: Ceres, 1976. 419p.

SOUZA, L.S. et al. Exigências edafo-climáticas. In: TRINDADE, A.V. (org.). Mamão. Produção: aspectos técnicos. Brasília: COMUNICAÇÃO PARA TRANSFERÊNCIA DE TECNOLOGIA, 2000. 77p. (Série Frutas do Brasil, 3).

SOUZA, J.M. et al. Biologia de Tetranychus mexicanus (McGregor) (Acari: Tetranychidae) em três espécies de Annonaceae. Neotropical Entomology, v.39, n.3, p.319-323, 2010. Disponível em: <http://www.scielo.br/pdf/ne/v39n3/ v39n3a02.pdf $>$. Acesso em: 05 set. 2011.

VAN DEN BOOM, C.E.M. et al. Differences among plant species in acceptance by the spider mite Tetranychus urticae Koch. Journal of Applied Entomology, v.127, p.177-183, 2003. 